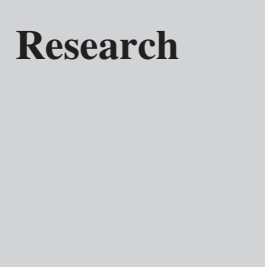

\title{
Shunt Types in Crystalline Silicon Solar Cells
}

\author{
O. Breitenstein ${ }^{*} \dagger$, J. P. Rakotoniaina, M. H. Al Rifai and M. Werner \\ Max Planck Institute of Microstructure Physics, Weinberg 2, D-06120 Halle, Germany
}

\begin{abstract}
Nine different types of shunt have been found in state-of-the-art mono- and multicrystalline solar cells by lock-in thermography and identified by SEM investigation (including EBIC), TEM and EDX. These shunts differ by the type of their I-V characteristics (linear or nonlinear) and by their physical origin. Six shunt types are process-induced, and three are caused by grown-in defects of the material. The most important process-induced shunts are residues of the emitter at the edge of the cells, cracks, recombination sites at the cell edge, Schottky-type shunts below grid lines, scratches, and aluminum particles at the surface. The material-induced shunts are strong recombination sites at grown-in defects (e.g., metal-decorated small-angle grain boundaries), grown-in macroscopic $\mathrm{Si}_{3} \mathrm{~N}_{4}$ inclusions, and inversion layers caused by microscopic SiC precipitates on grain boundaries crossing the wafer. Copyright (C) 2004 John Wiley \& Sons, Ltd.
\end{abstract}

KEY WORDS: shunts; thermography; lock-in; silicon; monocrystalline; multicrystalline

\section{INTRODUCTION}

$\Lambda$ solar cell, as simulated by essentially one-dimensional models, is assumed to show a homogeneous current flow across the whole area, both under illumination and in the dark. In the traditional interpretation of $I-V$ characteristics of solar cells all nonlinear currents belonged to the cell, and only ohmic current paths across the $p n$ junction have been attributed to 'shunts'. With the availibility of precision lock-in thermography techniques these shunts can be made visible, so in the following all bright features visible in thermography have been called 'shunts'. However, by later investigations it has turned out that there are not only ohmic shunts, but also diode-like ones, e.g., caused by local recombination sites. So the question, what is a shunt and what belongs to the undisturbed cell, has a philosophical dimension: can, e.g., a region of lower crystal quality be called a shunt? This question is still under discussion, but throughout this work we will use the term 'shunt' for any position in a solar cell showing under forward or reverse-bias a dark-current contribution additional to the diffusion current. In this sense edge leakage currents are shunting currents, but a region of lower crystal quality, where only the saturation current density of the diffusion current is increased, is not. Future discussions will show whether this definition will survive or has to be replaced by a more precise one.

\footnotetext{
*Correspondence to: O. Breitenstein, Max Planck Institute of Microstructure Physics, Weinberg 2, D-06120 Halle, Germany.

${ }^{\dagger}$ E-mail: breiten@mpi-halle.de

Contract/grant sponsor: BMWi; contract/grant number: 0329846 D (ASIS).

Contract/grant sponsor: EU; contract/grant number: ENK6-CT-2001-00573.
} 
The technique of infrared (IR) lock-in thermography, which has been commercially available for solar cell investigations since 2000, ${ }^{1}$ allows one to perform an efficient and systematic investigation of shunts in solar cells. ${ }^{2-5}$ This technique detects the periodic local surface temperature modulation in the positions of local shunts with a sensitivity below $100 \mu \mathrm{K}$ by applying a pulsed bias to the cell in the dark. Lock-in thermography may not only image the position of shunts up to an accuracy of $5 \mu \mathrm{m}$, but it also allows one to easily check whether shunts have a linear or a nonlinear $I-V$ characteristic, as well as to measure local $I-V$ characteristics quantitatively in a non-destructive way. ${ }^{3-6}$ However, knowing the positions of shunts and estimating their influence on the dark $I-V$ characteristic of the cell is only the first step in understanding shunt phenomena. The next step is to find out the physical nature of the shunts in order to avoid them in future. This can be done most successfully by using microscopic and microanalytic techniques such as EBIC (electron-beam-induced current) and EDX (energy-dispersive X-ray analysis) performed in the scanning electron microscope, TEM (transmission electron microscopy), and light microscopy. This contribution summarizes our efforts from the last 8 years to identify the physical origin of different shunt types in monocrystalline and multicrystalline silicon solar cells made by different producers. ${ }^{7}$

\section{EXPERIMENTAL}

The lock-in thermography investigations have been carried out partly with a home-made IR lock-in thermography system based on a $128 \times 128$ pixel InSb focal plane array thermocamera ${ }^{2,4}$ Amber AE 4128 , and partly with the commercial TDL 384 M 'Lock-in' thermography system made by Thermosensorik GmbH Erlangen, ${ }^{1,4,5}$ having a resolution of $384 \times 288$ pixel. For most IR investigations the sample was covered with a $20-\mu$ m-thin black-painted plastic film, which is sucked on to the cell by a vacuum and acts as an efficient IR emitter. For identifying the shunts, EBIC and EDX investigations were made on JEOL 6300/6400 scanning electron microscopes at acceleration voltages between 5 and $20 \mathrm{kV}$, which are equipped with an EBIC amplifier based on a DLPCA-200 variable gain current amplifier made by FEMTO and an EDX system IDFix made by SAMx. TEM investigations were made with a $1 \mathrm{MV}$ acceleration voltage on a JEOL 1000 high-voltage transmission electron microscope.

\section{RESULTS}

Before lock-in thermography allowed one to perform systematic shunt investigations, it was suspected that most shunts in multicrystalline silicon solar cells originated at crystal defects such as grain boundaries, precipitates, or dislocations. However, our investigations on hundreds of cells have revealed that by far most of the shunts are process-induced, whereas material-induced shunts are rather an exception than the rule. Moreover, in the past it was often assumed that shunts are generally characterized by a linear (ohmic) $I-V$ characteristic. However, we have found that in state-of-the-art silicon solar cells most shunts show a nonlinear (diode-like) $I-V$ characteristic. The linearity of the shunt characteristic can easily be checked by comparing lock-in thermograms taken at $0.5 \mathrm{~V}$ forward bias with thermograms taken at $-0.5 \mathrm{~V}$ reverse bias. If a shunt shows the same thermal signal under both conditions, its $I-V$ characteristic is linear. Otherwise, the characteristic is nonlinear. In silicon solar cells we have never found a case where the thermal signal was larger under low reverse bias than under the same forward bias. However, under large reverse bias of -5 to $-20 \mathrm{~V}$ new types of field-induced shunts may appear, especially in multicrystalline cells, even in positions, where no remarkable shunts have been detected under low-bias conditions. ${ }^{8}$ Therefore, shunt investigations performed under large reverse bias may be misleading for the shunting behavior at the working point of the cell. These field-induced shunts are beyond the scope of this contribution. Generally, it can be stated that linear shunts are more dangerous for degrading the performance of solar cells than nonlinear ones. In the following we will discuss the appearance of different processinduced and material-induced shunt types in lock-in thermography and present microscopic results supporting their physical interpretation. 


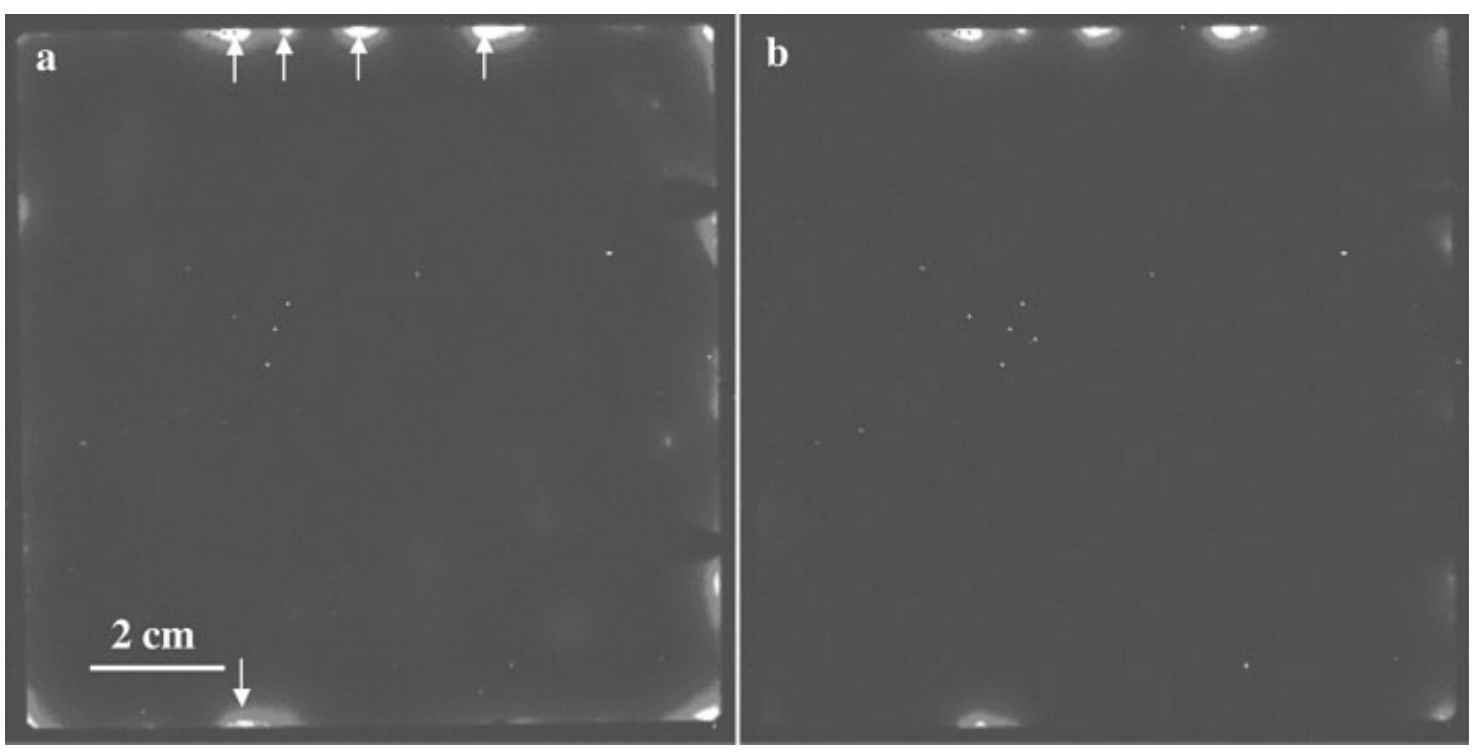

Figure 1. Lock-in thermogram of a cell containing edge shunts, measured under: (a) $+0 \cdot 5 \mathrm{~V}$; (b) $-0 \cdot 5 \mathrm{~V}$ bias

\section{Process-induced shunts}

\section{Linear edge shunts}

This was the dominant shunt type in earlier solar cell technologies, but with improving edge isolation technologies it appears less frequently now. Figure 1 shows a lock-in thermogram of a cell measured at $0.5 \mathrm{~V}$ forward bias compared with one measured at $-0.5 \mathrm{~V}$ reverse bias, both scaled from $0 \mathrm{mK}$ T-modulation amplitude (black) to $1 \mathrm{mK}$ (white). The comparison of the brightness of the shunts measured under both conditions shows that only some of the edge shunts marked by arrows in Figure 1 are ohmic.

In order to investigate their nature, EBIC investigations have been performed in the edge region in shunt positions. Figure 2 shows such an EBIC image, together with the corresponding secondary electron (SE)

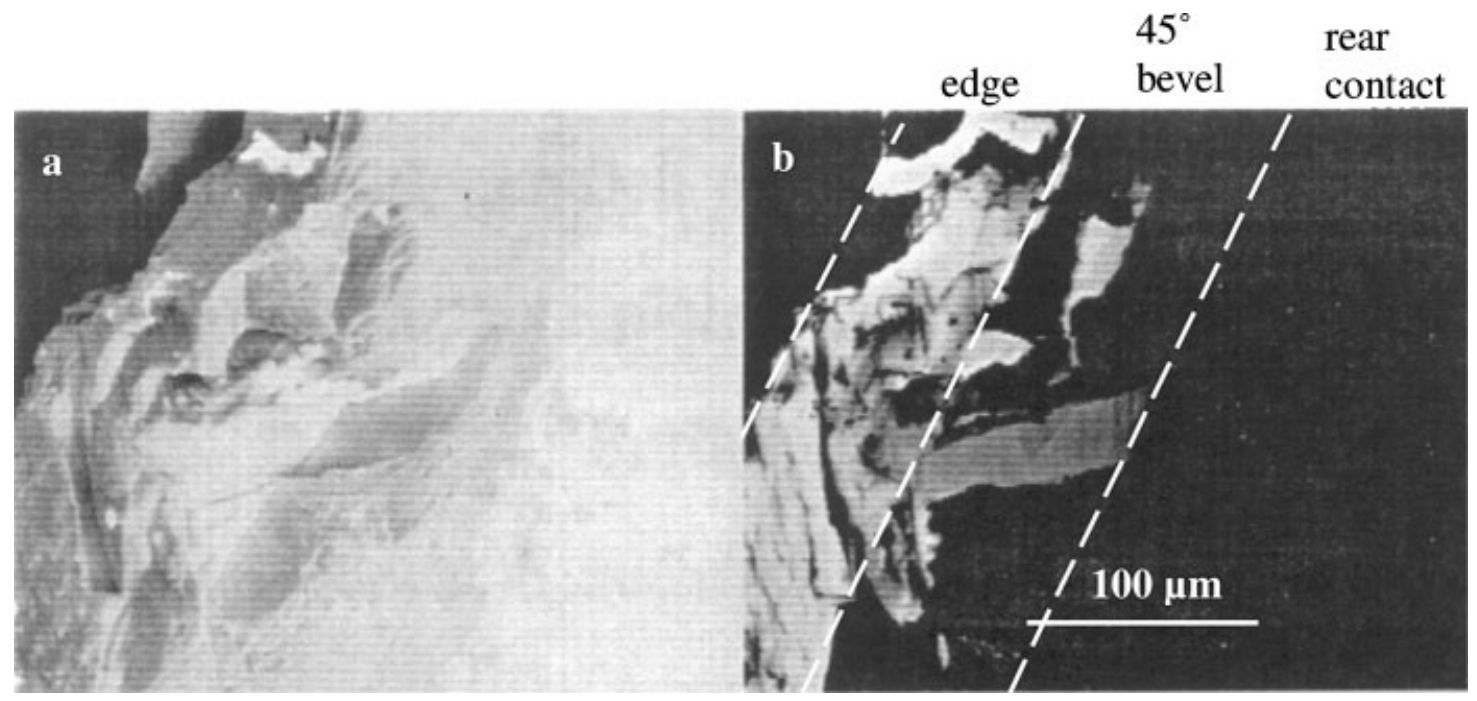

Figure 2. (a) SE image; (b) EBIC image at the position of a linear edge shunt. Image taken at an angle of $45^{\circ}$ from the rear side 
image. ${ }^{6}$ We are looking at an angle of approximately $45^{\circ}$ from the rear to the edge of the cell. Here the $p n$ junction was opened by grinding a $45^{\circ}$ bevel at the rear edge, which actually should show no EBIC signal. In this bevel we see a horizontal stripe where an EBIC signal linearly decreases from a maximum value at the emitter (left) to zero at the back contact. Such a behavior is expected for a pn junction, which is shortcircuited at one side. The stripe is a remainder of the emitter, which was not 'opened' by the edge insulation procedure. So linear edge shunts are usually due to an incompletely opened emitter at the edge.

\section{Nonlinear edge shunts}

Those edge shunts which appear in Figure 1 predominantly under forward bias $(+0.5 \mathrm{~V})$, show a nonlinear (diode-like) $I-V$ characteristic. These shunts can be interpreted to be recombination sites, which are acting at the edge where the $p n$ junction crosses the surface. If the recombination activity of these surface states remains low, their recombination current has an exponential characteristic with an ideality factor of 2 , as expected from Shockley diode theory. ${ }^{9}$ However, many of these shunts show an exponential characteristic with an ideality factor of 3 and above. ${ }^{3}$ These large ideality factors seem to be a general property of nonlinear local shunts, since we have measured them very often. The physical mechanism leading to large ideality factors is not clear yet.

\section{Cracks and holes}

It was shown that cracks in readily processed solar cells lead to a weak nonlinear edge recombination current, similar to nonlinear edge shunts. ${ }^{2}$ However, if a crack is already present in the wafer before processing, or if it appears during processing before finally screen-printing the contact metallization, cracks may lead to severe ohmic shunts. If the crack is already present in the raw wafer, an emitter layer may be established across the crack. This layer shorts the emitter against the base contact, hence a linear (ohmic) shunt appears along the crack, acting like a linear edge shunt. Figure 3 shows an example of such a crack-induced shunt at the lower edge of a cell. The maximum T-modulation amplitude measured in Figure 3, which is a measure of the current flowing in this individual shunt, was in the order of $10 \mathrm{mK}$. However, since this contribution deals rather with the qualitative description of different shunt types than with their quantitative description, ${ }^{5}$ the T-modulation amplitudes are not given for all thermograms of this contribution. Note that the weak vertical bright stripe above the crack is the electrical connection from the major grid to the crack shunt, which becomes thermographically visible by Joule heating. If during screen-printing some metal paste penetrates a crack, after firing this may

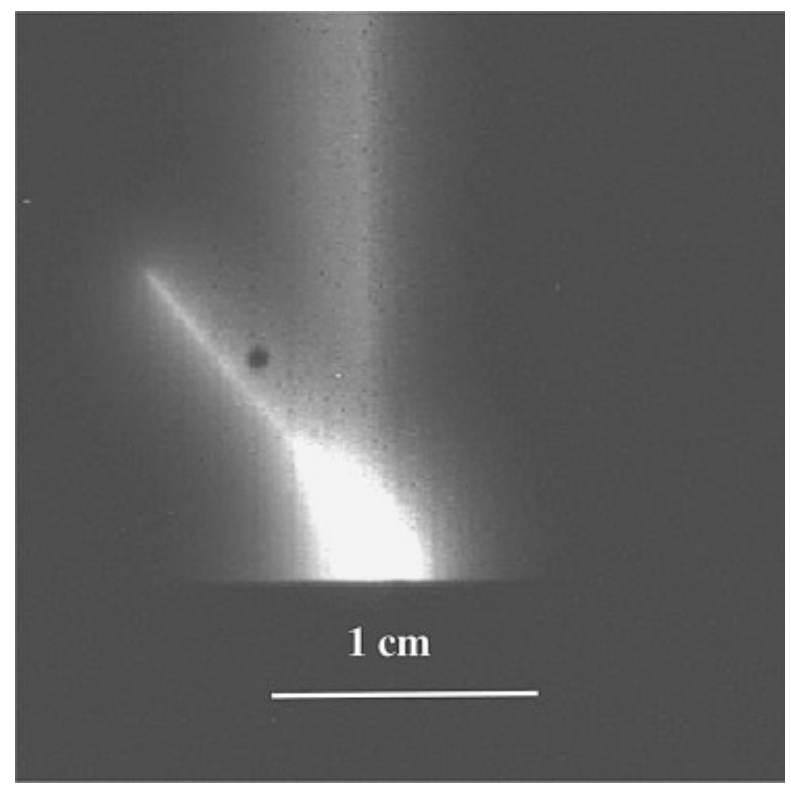

Figure 3. Lock-in thermogram of a shunt caused by a crack at the lower edge of a cell 

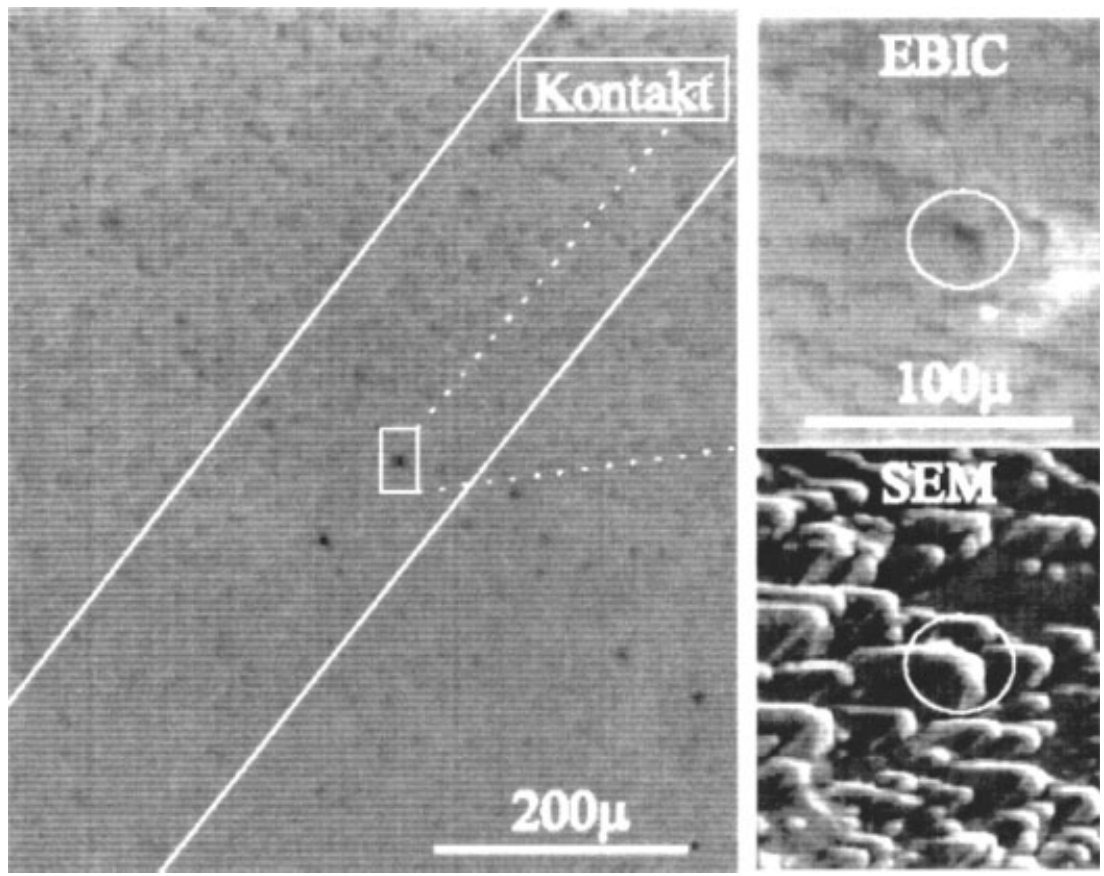

Figure 4. EBIC and SE images of a shunt below a grid line

produce especially strong linear shunts. The shunts described above may also emerge if there are any holes present in a cell, e.g., coming from laser cutting.

\section{Schottky-type shunts}

In order to have a good blue response, the emitter of silicon solar cells has a thickness in the order of only $0 \cdot 3 \mu \mathrm{m}$. If the emitter metallization is sintered, it consumes some silicon material. If the sintering parameters are not optimized to the emitter doping profile, the emitter metallization may punch through the emitter, finally leading to a Schottky-type direct contact between the metal and the $p$-type base material. As a rule, this contact has a more or less rectifying characteristic, but it is far away from an ideal Schottky diode. Just as for Schottky diodes containing some interface layer, its ideality factor is not unity, but may be even larger than two. Schottky-type shunts may also appear if the emitter metallization is printed at a position having no emitter layer at all. Such 'holes' in the emitter may be due to mechanical violation of texturization pyramide tips after emitter doping or due to dust particles or insufficient contact of the phosphorus glass to the silicon in some positions. Figure 4 shows two EBIC and one SEM image of a region below a grid finger (region between the two lines: Kontakt) in the position of a nonlinear shunt after dissolving the metal with HF. ${ }^{6}$ The dark spot in EBIC is a mechanically violated tip of a large texturization pyramid, where the emitter is missing. In rare cases ohmic shunts were also found below grid lines, the nature of which is still unclear.

\section{Scratches}

A scratch at the surface of a solar cell across the emitter layer brings the pn junction to a surface with a high density of recombination centers. Thus, scratches act like nonlinear edge shunts, leading typically to an exponential characteristic with a large ideality factor, like nonlinear edge shunts. Figure 5 shows a lock-in thermogram of a cell containing two scratches, which were only visible under forward bias. If scratches are made before emitter diffusion, or if they do not penetrate the $p n$ junction, they generate local recombination centers, also acting as nonlinear shunts, similar to strongly recombination-active grown-in crystal defects (see below).

\section{Aluminum particles}

Whenever aluminum reaches the front surface of a cell before firing, which may happen, e.g., by cross-contamination during stacking of the cells, it will create shunts. During firing Al will alloy in, leading to a $p^{+}$-doped 


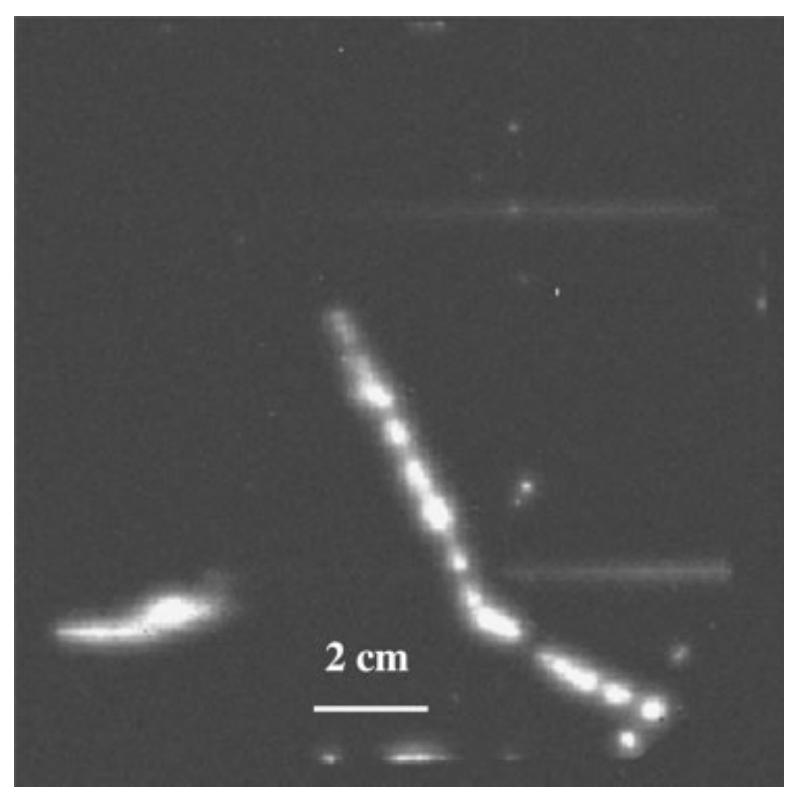

Figure 5. Lock-in thermogram of a cell with two scratches

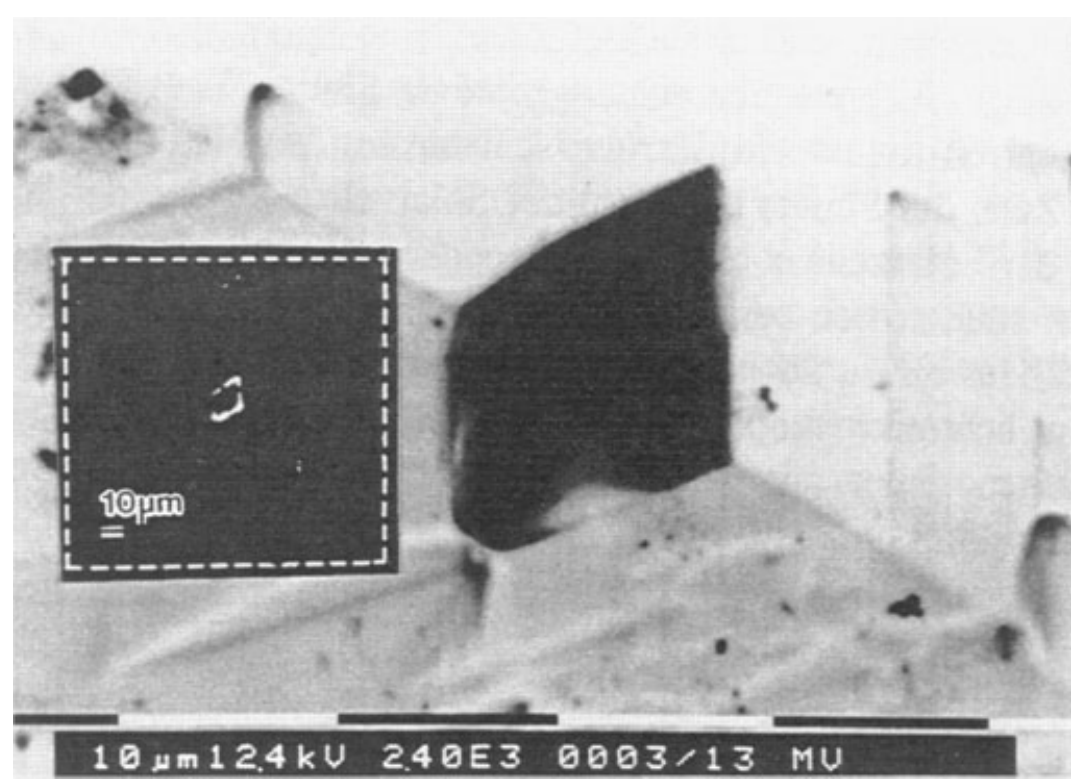

Figure 6. EBIC image of the position of an Al-induced shunt after etching away the Al particle. Insert: light emission at $-10 \mathrm{~V}$ reverse bias, light microscope image

region around the particle, which may compensate the emitter. This $p^{+}$-region may be in ohmic contact with the base, but it also makes a tunnel contact to the emitter, thus producing a shunt. Figure 6 shows an EBIC image of the position of such a shunt, after the $\mathrm{Al}$ has been dissolved by $\mathrm{HF}$ (H. Gottschalk, Cologne, personal communication of work from Project 0329536A-I, DIXSI). The $10 \mu \mathrm{m}$ rhombohedral Al particle (identified by EDX) was sitting on a twin grain boundary. Its former position appears dark in EBIC, hence there is no pn junction anymore, as expected. Interestingly, after applying a reverse bias of $-10 \mathrm{~V}$ a visible luminescence appeared at the edge of this region (see light microscope image, insert in Figure 6). This is obviously an avalanche breakdown, which Gottschalk has also found occasionally in other shunt locations. 

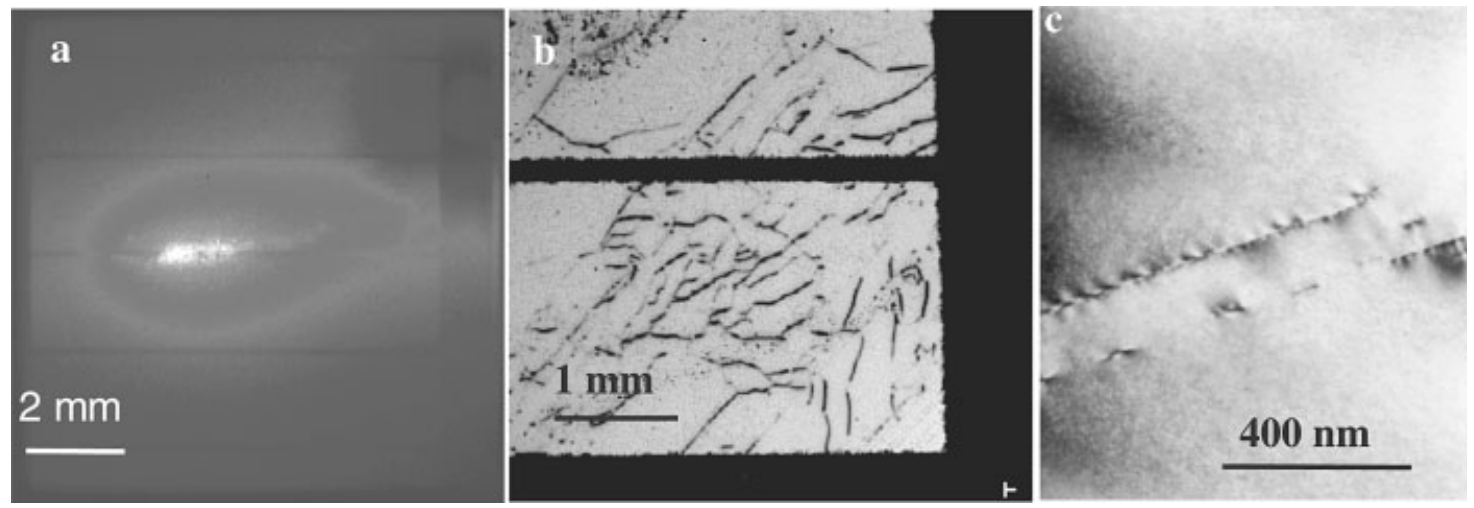

Figure 7. (a) Lock-in thermogram; (b) EBIC image; (c) and TEM image of a shunt caused by strongly recombinative crystal defects

\section{Material-induced shunts}

\section{Strongly recombinative crystal defects}

It had been mentioned that ordinary grain boundaries, which are easily visible in LBIC or EBIC due to their recombination activity, do not usually lead to measurable shunts. However, if a crystal defect is strongly recombinative, its recombination current may become strong enough for it to act as a shunt, similar to nonlinear edge shunts and scratches. Figure 7 (a) shows a lock-in thermogram of one of these shunts, which are visible only under forward bias. ${ }^{10}$ The EBIC image in this region (Figure $7 \mathrm{~b}$ ) showed a network of dark lines, which turned out in TEM (Figure 7c) to be small-angle grain boundaries. It should be mentioned that the TEM investigation did not reveal any precipitates at these dislocations. Nevertheless, they should be decorated by impurities, otherwise they would not be so recombinative. Similar shunts have also been found within regions of poor crystal quality (dislocation tangles). ${ }^{6}$ Note, however, that the general increase of the dark forward current in regions of lower crystal quality, leading to a 'cloudy' appearance of the thermograms showing a correlation to LBIC, cannot be interpreted as shunts. Instead, these are inhomogeneities of the diffusion current owing to the lower diffusion length in these regions, which can be proved by thermographic ideality factor mapping. ${ }^{3}$

\section{Macroscopic $\mathrm{Si}_{3} \mathrm{~N}_{4}$ inclusions}

Occasionally, cast multicrystalline silicon material contains macroscopic inclusions, which can be identified by EDX as $\mathrm{Si}_{3} \mathrm{~N}_{4}$. If a solar cell is processed from such a wafer, the inclusion is easily visible as a grey region, seemingly without ARC. In reality the $\mathrm{Si}_{3} \mathrm{~N}_{4} \mathrm{ARC}$ is just invisible there, since it has the same optical properties as the inclusion. TEM investigations and EDX have revealed that the inclusion consists of amorphous $\mathrm{Si}_{3} \mathrm{~N}_{4}$ containing 100-200 nm crystalline $\mathrm{Si}_{3} \mathrm{~N}_{4}$ particles. Figure 8 shows a lock-in thermogram of a cell containing a $\mathrm{Si}_{3} \mathrm{~N}_{4}$-induced shunt (arrow). Also this shunt was visible only under forward bias, hence it has a nonlinear $I-V$ characteristic. We assume that the recombination activity of interface states between $\mathrm{Si}$ and this $\mathrm{Si}_{3} \mathrm{~N}_{4}$ material is responsible for these shunts. Most probably the $\mathrm{Si}_{3} \mathrm{~N}_{4}$ particles stem from the walls of the crucible used for crystallizing the Si material.

\section{Inversion layers at precipitates}

A significant amount of multicrystalline silicon cells showing a very low parallel resistance leads to lock-in thermograms, showing a network of strong linear (ohmic) shunts like those shown in Figure 9. The positions of these shunts often correlate with certain large-angle grain boundaries in the material. These shunts are also visible in EBIC as dark clouds around the grain boundaries (Figure 10a). In order to investigate the nature of these shunts, the rear contact and the highly $p$-doped back-surface field (BSF) layer have been removed by subsequent etching with $\mathrm{HCl}$ and $\mathrm{HF} / \mathrm{HNO}_{3}$. Then EBIC investigations have been performed in the shunt regions, both at the front and at the rear side. In the front side EBIC image the dark clouds have now disappeared. Figure 10 (b) shows an EBIC image taken from the rear side. It shows a number of bright lines with a collection 


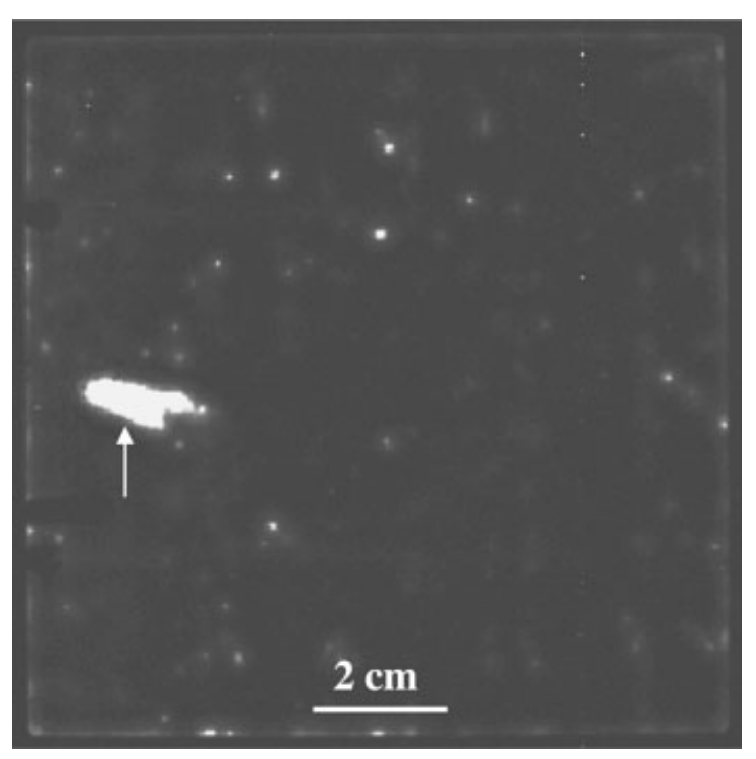

Figure 8. Lock-in thermogram of a cell containing a $\mathrm{Si}_{3} \mathrm{~N}_{4}$ inclusion (arrow)
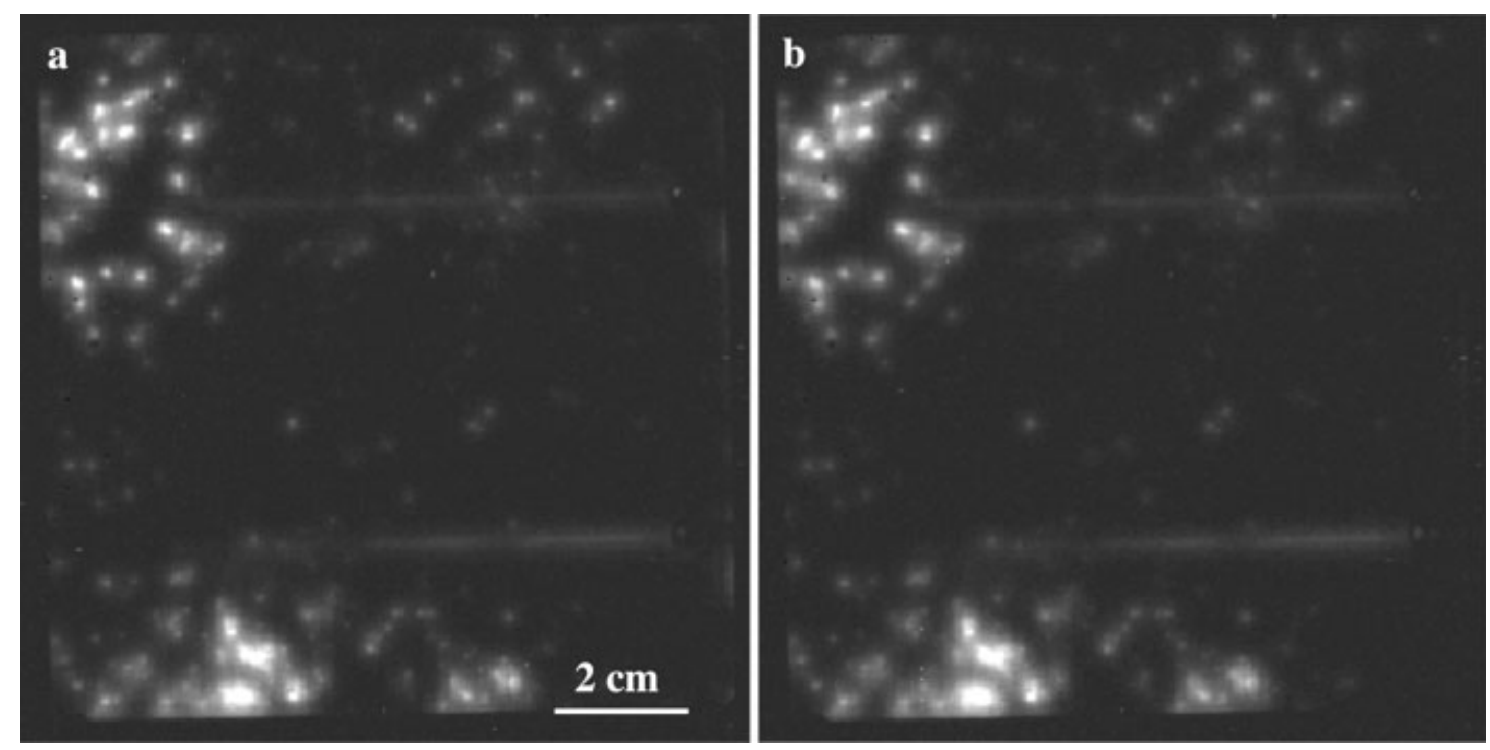

Figure 9. Lock-in thermogram of a strongly shunted cell at: (a) $0.5 \mathrm{~V}$ forward; (b) $-0.5 \mathrm{~V}$ reverse bias

efficiency close to 1 in positions of large-angle grain boundaries. These lines can be explained only as carriercollecting inversion channels crossing the wafer, which are connected to the emitter. Inversion means a local transformation of the silicon material from $p$ - to $n$-type, caused by the presence of fixed positive charges in the corresponding grain boundaries. In the complete solar cell these channels are electrically connected with the emitter and may also be connected with the rear contact, thereby shorting the cell in this position. For this contact the BSF plays a major role, since the inversion channels become visible in rear-side EBIC only after dissolving the BSF layer. After this investigation a plane view TEM specimen was prepared in the position of one channel (arrow in Figure 10). Figure 11 shows a TEM overview image, composed from several single images, of this grain boundary. We see a number of large crystalline precipitates up to $5 \mu \mathrm{m}$ in size with a mean distance up to $15 \mu \mathrm{m}$, embedded in this grain boundary. According to selected area diffraction and EDX investigations 


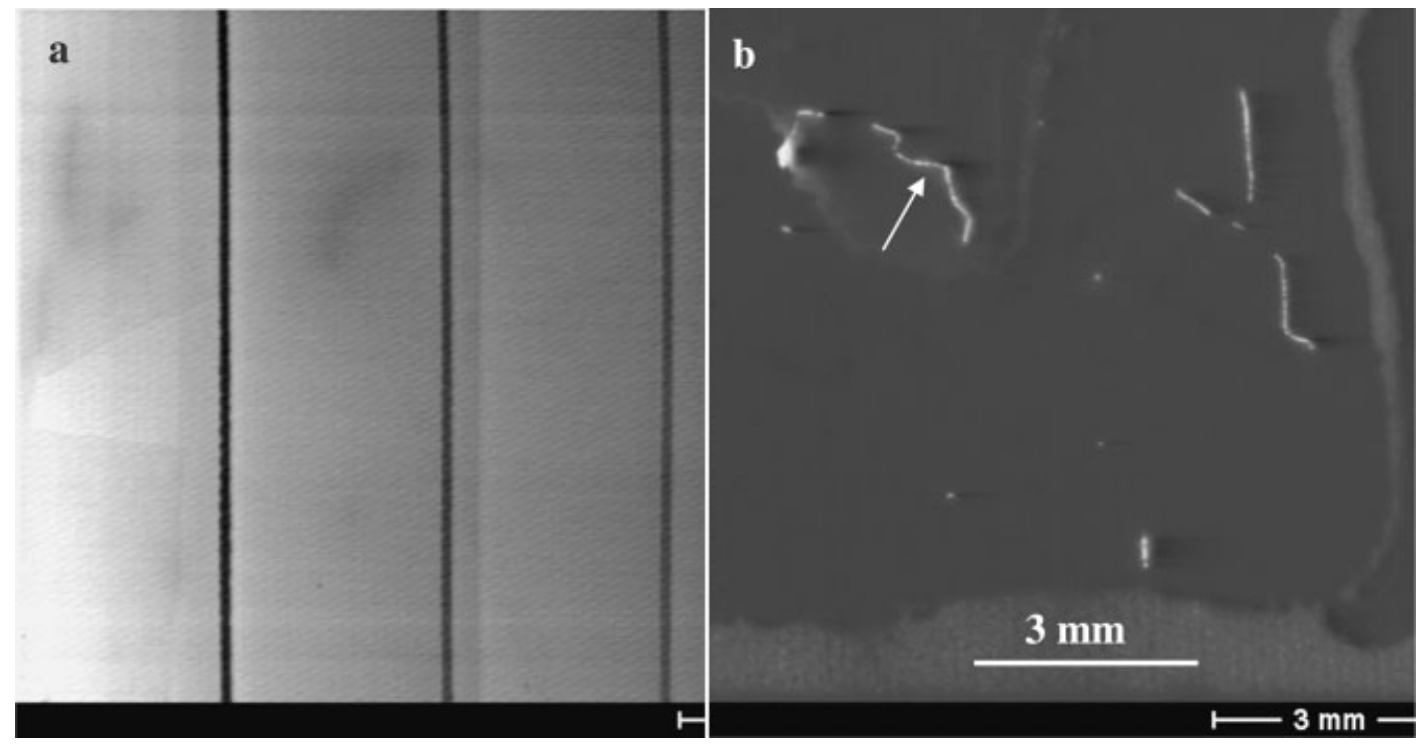

Figure 10. (a) EBIC image of a shunt region; (b) the same region after removing the rear contact and the BSF layer, imaged from the rear

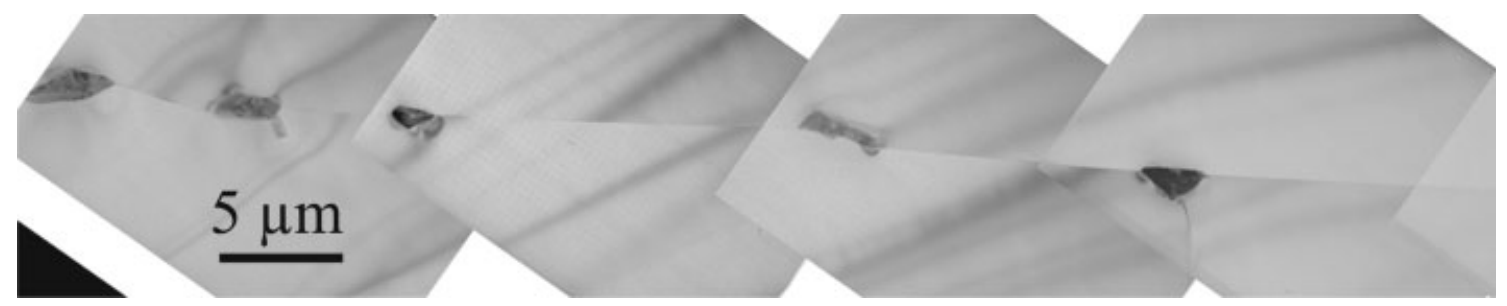

Figure 11. TEM image of SiC precipitates at a large-angle grain boundary inducing an inversion layer

these precipitates consist of cubic SiC. No such precipitates ever have been found in grain boundaries of solar cells not showing this type of shunts. So the precipitates should be physically connected with the formation of the inversion layers along these grain boundaries. However, since according to Figure 11 these particles do not overlap, the physical origin of the vertical current flow across the cell is not clear yet. Similar precipitates have already been found in a similar shunt in a cell made by another producer, ${ }^{10}$ hence this type of shunt is obviously not very rare. It can be classified as the most dangerous material-induced shunt type in multicrystalline silicon solar cells.

\section{CONCLUSIONS}

After lock-in thermography enabled the systematic investigation of shunts in silicon solar cells also under forward bias conditions, the physical understanding of shunts has greatly improved. Since the shape of the $I-V$ characteristic is an important characteristic of the nature of a shunt, a key experiment for identifying shunts is the comparison of lock-in thermograms taken under the same forward and reverse bias. This information, together with that about the position of a shunt, usually allows one to classify most shunts into one of the categories listed in this contribution. A linear shunt at the edge is usually an incompletely opened emitter, and a linear shunt within the area is a good candidate for an inversion channel, if it is not lying on a crack or a hole. Shunts with a nonlinear characteristic are usually recombination-induced shunts caused by surface or interface states or by strongly recombinating crystal defects. If nonlinear shunts are below grid lines, they may be of 
Schottky type. In both cases the ideality factor of their characteristic is often significantly larger than 2, which is not completely understood yet. However, it seems to be clear that the often observed 'second diode' current with a large ideality factor is usually due to the existence of these nonlinear shunts., 311

\section{Acknowledgements}

The authors are grateful to M. Langenkamp (Rendsburg), I. Konovalov (Leipzig), S. Neve (Hannover), and H. Gottschalk (Cologne) for experimental cooperation and to S. Lust (Q CELLS AG, Thalheim), Q. N. Lê (Photowatt SA, Bougoin-Jallieu), and T. Bruton (BP Solar Ltd, Sunbury) for delivering cells for these investigations. This work was supported by BMWi Project 0329846 D (ASIS) and by EU Project ENK6-CT-200100573 (PORTRAIT).

\section{REFERENCES}

1. www.thermosensorik.de

2. Breitenstein O, Langenkamp M, Lang O, Schirrmacher A. Shunts due to laser scribing of solar cells evaluated by highly sensitive lock-in thermography. Solar Energy Materials and Solar Cells 2001; 65: 55-62.

3. Breitenstein O, Langenkamp M, Rakotoniaina JP, Zettner J. The imaging of shunts in solar cells by infrared lock-in thermography. Proceedings of the 17th European Photovoltaic Solar Energy Conference, Munich, 2002; 1499-1502.

4. Breitenstein O, Langenkamp M. Lock-in Thermography-Basics and Application to Functional Diagnostics of Electronic Components, Springer Series Advanced Microelectronics, Volume 10. Springer: Heidelberg, Berlin, 2003, ISBN 3-540-43439-9.

5. Breitenstein O, Rakotoniaina JP, Al Rifai MH. Quantitative evaluation of shunts in solar cells by lock-in thermography. Progress in Photovoltaics: Research and Applications 2003; 11: 512-526.

6. Konovalov I. Zusammenhang zwischen elektrischen Verlustmechanismen und der Struktur von Solarzellen. PhD. Thesis, Martin Luther University, Halle, 2000.

7. Breitenstein O, Rakotoniaina JP, Neve S, Al Rifai MH, Werner M. Shunt types in multicrystalline solar cells. Proceedings of the 3rd World Conference on Photovoltaic Energy Conversion, Osaka, 2003; 987-990.

8. Breitenstein O, Rakotoniaina JP, Schmidt J. Comparison of shunt imaging by liquid crystal sheets and lock-in thermography. Proceedings of the 12th Workshop on Crystalline Solar Cell Materials and Processes, Breckenridge, Colorado, 2002; 244-247.

9. Kühn R, Fath P, Bucher E. Effects of pn-junctions bordering on surfaces investigated by means of 2D-modeling. Proceedings of the 28th IEEE PVSC, Anchorage, 2000; 116-119.

10. Rakotoniaina JP, Neve S, Werner M, Breitenstein O. Material-induced shunts in multicrystalline silicon solar cells. Proceedings of the Conference on PV in Europe, Rome, 2002; 24-27.

11. Breitenstein O, Rakotoniaina JP, Neve S, Green MA, Zhao J, Wang A, Hahn G. Lock-in thermography investigation of shunts in screen-printed and PERL solar cells. Proceedings of the 29th IEEE PVSC, New Orleans, 2002 ; $430-433$. 